\title{
Transtornos psiquiátricos menores e procura por cuidados em estudantes de Medicina
}

\author{
Minor psychiatric disorders and the search for \\ care by medical students
}

\author{
Marco Antonio Buch Cunha \\ Antonio Augusto de França Neves ${ }^{\mathrm{I}}$ \\ Maria Elisa Moreira ${ }^{\mathrm{I}}$ \\ Fernanda Jacopucci Hehn ${ }^{\text {II }}$ \\ Tatiana Paludetto Lopes ${ }^{\mathrm{I}}$ \\ Carolina Cruz Furtado Ribeiro ${ }^{\text {III }}$ \\ Ágnes de Paula Fernanda Watanabe ${ }^{\mathrm{IV}}$
}

\section{PALAVRAS-CHAVE \\ - Saúde mental \\ - Estudantes de medicina \\ - Automedicação}

Recebido em: 21/07/2008 Reencaminhado em: 12/11/2008 Aprovado em: 27/11/2008

REVISTA BRASILEIRA DE EDUCAÇÃO MÉDICA

\section{KEY WORDS}

- Medical students

- Self medication

\begin{abstract}
RES U M O
A carreira médica pode desencadear alterações patológicas na saúde mental, que podem ter início já na graduação. O objetivo deste estudo foi identificar a prevalência de Transtornos Psiquiátricos Menores (TPMe) e a procura por ajuda em estudantes de um curso de Medicina. Trata-se de um estudo transversal, com uma população de 343 estudantes da primeira à quarta série, maiores de 18 anos. Foram realizadas entrevistas, em salas de aula, por meio de dois questionários estruturados, o Self Reporting Questionnaire (SRQ-20) e outro elaborado pelos autores. Análises descritiva, univariada, bivariada e estatística foram utilizadas mediante o programa Microsoft Excel. Os resultados evidenciaram que, entre os acadêmicos com TPMe, 41,6\% (a maioria) moravam sozinhos e 75\% eram mulheres. Entre os acometidos, 59,2\% não conheciam qualquer programa e apenas 9,1\% procuraram ajuda. O uso de medicamento foi duas vezes mais prevalente em mulheres com TPMe do que em homens, sendo antidepressivos e ansiolíticos os mais usados. A prevalência de 26,1\% de TPMe nos alunos, associada a baixa procura por cuidados e a relatos de automedicação, demonstra a inefetividade dos atuais programas de apoio.
\end{abstract}

\section{A B S T R A C T}

The medical career can trigger pathological changes in mental health that may even begin during undergraduate medical training. The purpose of this study was to identify the prevalence of minor psychiatric disorders (MPD) and the search for counseling by medical students. This was a cross-sectional study of a sample of 3431 st to $4^{\text {th }}$-year medical students 18 years and older. Interviews were conducted in the classroom using two structured questionnaires, the Self Reporting Questionnaire (SRQ-20) and another prepared by the authors. Descriptive, univariate and bivariate analyses were performed using Microsoft Excel. Among students with MPD, 41.6\% lived alone and 75.0\% were women. Among the ill, 59.2\% were unfamiliar with any counseling program, and only $9.1 \%$ sought help. Medication for PMD was twice as prevalent in females as compared to males (with antidepressants and anxiolytics as the most frequently used medicines). The $26.1 \%$ prevalence rate of students with MPD, plus the frequent self-medication and low demand for professional care, demonstrated the ineffectiveness of current psychological support programs.

\footnotetext{
I Universidade de Taubaté, Taubaté, São Paulo, Brasil.

${ }^{I I}$ Hospital Beneficência Portuguesa, São Paulo, São Paulo, Brasil.

II Hospital CEMA, São Paulo, São Paulo, Brasil.

IV Santa Casa de Misericórdia de São Paulo, São Paulo, São Paulo, Brasil.
} 


\section{INTRODUÇÃO}

Há inúmeras gratificações psicológicas inerentes à profissão médica. Aliviar a dor e o sofrimento, curar doenças, salvar vidas, diagnosticar corretamente, sentir-se competente, ensinar, aconselhar, educar, prevenir doenças, receber reconhecimento e gratidão são algumas das características psicológicas da tarefa médica que fazem da medicina uma profissão ainda muito atraente e gratificante.

Ainda que atrativa, sabe-se que a carreira médica pode desencadear desequilíbrio emocional com consequências patológicas para a saúde mental, tendo início ainda na graduação do ensino médico.

Quando o estudante não consegue elaborar os conflitos gerados pelas dificuldades próprias do curso médico, é importante que existam propostas de assistência psicológica e acompanhamento psicopedagógico como parte de um programa de atenção primária à saúde dos estudantes.

O Programa de Apoio ao Estudante (PAE), desenvolvido na universidade em que este estudo foi realizado, tem como princípio minimizar os conflitos internos dos estudantes da comunidade acadêmica e, como objetivo, orientar e apoiar todos os estudantes em sua adaptação no meio universitário, além de ajudar o aluno e seus familiares em situações de crise que interfiram nas questões psicopedagógicas ${ }^{1}$.

O Departamento de Medicina tem também, há sete anos, um serviço de assistência aos alunos com dificuldades pedagógicas e afetivas, com atribuição de horas-aula à disciplina de Psiquiatria. Além disso, o curso de Medicina desta universidade apresenta a disciplina Psicologia Médica na grade curricular.

Nos últimos quatro anos, a chefia do Departamento e a coordenadora de ensino do curso de Medicina propuseram o Projeto Tutoria, por meio do qual alunos com dificuldades pedagógicas e comportamentais são encaminhados para acompanhamento por professores voluntários.

É essencial que esteja disponível uma estrutura acadêmica formal que forneça apoio psicopedagógico e emocional efetivo a seus alunos para lidar com situações trágicas como o suicídio e, principalmente, para evitar que um ato como este possa ocorrer.

A raiva, o medo, a culpa, a tristeza, a ansiedade são alguns dos sentimentos contraditórios gerados com o suicídio de um integrante de um grupo, como o dos estudantes de Medicina.

A motivação do grupo para a pesquisa surgiu de um caso de suicídio ocorrido na universidade, que suscitou questionamentos sobre a saúde mental dos acadêmicos e sobre os cuidados disponíveis.
O desenvolvimento de estudos e pesquisas sobre a saúde psicológica do estudante de Medicina e do médico, portanto, é relevante e necessário.

\section{REVISÃO DA LITERATURA}

A importância da saúde mental é reconhecida pela Organização Mundial de Saúde (OMS) desde a sua origem, o que se reflete em sua definição de saúde não como simplesmente a ausência de doença ou enfermidade, mas como "um estado de completo bem-estar físico, mental e social". Nos últimos anos, esta definição ganhou maior destaque, como resultado de muitos progressos nas ciências biológicas e comportamentais. Estes, por sua vez, contribuíram para o aperfeiçoamento da maneira de compreender o funcionamento mental e a profunda relação entre saúde mental, física e social. Desta nova concepção emerge uma nova esperança. Para todas as pessoas, a saúde mental, a saúde física e a social são fios da vida estreitamente entrelaçados e profundamente interdependentes. À medida que cresce a compreensão desse relacionamento, torna-se cada vez mais evidente que a saúde mental é indispensável ao bem-estar geral dos indivíduos, das sociedades e dos países²

Atualmente, algumas faculdades de Medicina ainda negligenciam o envolvimento emocional dos alunos em relação ao sofrimento dos pacientes, sendo que durante a formação médica os estudantes estão expostos a emoções negativas, como angústia, frustração e medo. Assim, gera-se um profissional treinado para diagnosticar doenças e restabelecer a saúde, mas não para enfrentar a morte e o morrer ${ }^{3}$.

No tocante à formação acadêmica em particular, sabe-se que o modelo de ensino médico ficou estagnado por várias décadas e somente nos últimos anos tem se deparado com a necessidade crescente de reformas curriculares e pedagógicas.

Alguns fatores estressantes, como pressão para aprender, grande quantidade de novas informações e falta de tempo para atividades sociais, também contribuem para desencadear distúrbios emocionais nessa população ${ }^{4}$. Há relatos de perda da liberdade pessoal, sentimentos de desumanização e forte competição entre os colegas 5 .

A questão da saúde mental dos universitários tem sido alvo de estudos em diversos países, envolvendo, sobretudo, estudantes de Medicina, que estão sujeitos a diversos fatores de estresse que poderão desencadear distúrbios emocionais, como depressão, angústia, isolamento, irritabilidade, ou distúrbios físicos, como gastrite, diarréia, cefaléia e emagrecimento ${ }^{6}$.

Esses sinais e sintomas fazem parte dos TPMe, que incluem os seguintes subgrupos de diagnósticos: distúrbios afetivos (de- 
pressão, distimia); distúrbios de ansiedade (estados generalizados de ansiedade, distúrbios pós-traumáticos agudos e crônicos, estados de ansiedade atípica); distúrbios fóbicos (agorafobia, fobias sociais e simples); distúrbios de somatização (distúrbios conversivos e dissociativos, síndrome de somatização, dor psicogênica $)^{7}$.

Segundo Costa e Pereira ${ }^{6}$, foi diagnosticada depressão em 23\% dos estudantes de Medicina da Universidade do Mississipi, $57 \%$ deles com altos níveis de desconforto somático. No Brasil, numa universidade privada, a prevalência dos sintomas depressivos nos estudantes de Medicina foi de 40,7\%5; noutra instituição estadual, a prevalência foi cerca de quatro vezes maior, em média, do que na população geral americana e mais do que sete vezes na população brasileira ${ }^{8}$.

Um estudo realizado por uma universidade federal apontou que a depressão atinge 9,3\% dos indivíduos no primeiro ano de residência médica. Em outros países, estudos sugerem uma incidência de até $30 \%$. Nessa mesma universidade foi criado o Serviço de Apoio Psicopedagógico (Sapa), que se propõe a estar atento a questões emocionais e psicossociais e a atuar nesta área, procurando examinar e orientar os alunos em suas eventuais dificuldades no trabalho de aprendizagem. Proporciona-se ao aluno atendimento psiquiátrico e psicoterápico individual, num referencial de psicoterapia breve ${ }^{9}$.

Outro serviço de apoio foi desenvolvido por uma universidade estadual em 1986: o Grupo de Assistência Psicológica ao Aluno (Grapal). As atividades desse serviço consistem em: entrevistas com alunos do primeiro ano, grupos de reflexão com alunos do quinto ano sobre o papel do médico, psicoterapia, atendimento clínico, orientação familiar e aos professores ${ }^{10}$.

Os locais de assistência especializada, no entanto, ainda são poucos no Brasil, e só recentemente o tema passou a ser mais discutido $^{9}$.

\section{OBJETIVOS}

Identificar a prevalência de sintomas de transtornos psiquiátricos menores em estudantes de medicina de uma universidade do Vale do Paraíba e a procura por cuidados durante a graduação.

\section{CASUÍSTICA E MÉTODOS}

Trata-se de um estudo transversal, realizado no Departamento de Medicina de uma universidade situada em um município do Vale do Paraíba. A população do estudo abrangeu 343 estudantes do curso de Medicina, matriculados da primeira à quarta série, sendo excluídos os menores de 18 anos.
A coleta de dados foi realizada por meio de entrevistas, em salas de aula e antes do início da aula, apoiadas na aplicação de dois questionários estruturados, o SRQ 20 - SelfReporting Questionnaire, desenvolvido por Harding et al. ${ }^{11}$ e validado por uma série de estudos internacionais conduzidos pela OMS, como um instrumento para o screening dos TPMe não psicóticos. Sua utilidade foi comprovada para identificar casos de alterações na saúde mental. O ponto de corte deste questionário foi de oito ou mais respostas "sim" no $S R Q-20$ como indicador de prováveis casos de transtornos psiquiátricos menores ${ }^{12}$ (APÊNDICE A).

O questionário desenvolvido pelos autores desta pesquisa inclui características demográficas dos acadêmicos, conhecimento em relação aos serviços de assistência psicossocial oferecidos pela universidade, bem como a procura por cuidados nestes serviços e em outros disponíveis na comunidade.

Como contrato bioético, foi elaborada a carta de apresentação ao responsável pelo curso de Medicina e a carta de consentimento livre e esclarecido, garantindo-se participação voluntária, confidencialidade, sigilo e retorno dos resultados da pesquisa aos interessados, bem como encaminhamento dos casos que solicitaram ajuda para apoio psicopedagógico.

Foram realizadas análises descritiva, univariada, bivariada e estatística dos dados, pelo teste de qui quadrado e correção de Yates para $\mathrm{p}<0,05$, por meio do programa Microsoft Excel.

Este estudo foi aprovado pela Comissão de Ética e Pesquisa da Universidade de Taubaté sob o número 0181/07.

\section{APRESENTAÇÃO E DISCUSSÃO DOS RESULTADOS}

\section{Análise descritiva}

Do total de 343 alunos matriculados da primeira à quarta série do curso de Medicina, 295 (86,0\%) foram entrevistados. Os motivos pelos quais $48(14,0 \%)$ alunos não participaram da pesquisa foram: ausência no dia da entrevista, ter menos de 18 anos e não adesão.

Destes 295 alunos, 121 (41,9\%) eram do sexo masculino e 168 $(57,0 \%)$ do sexo feminino, sendo que $6(2,0 \%)$ não responderam ao item gênero. Estes dados coincidem com a maior procura do sexo feminino pelo curso de Medicina, o que também foi encontrado numa universidade estadual a partir de 1995, sendo que, em 2003, 64,4\% dos alunos eram mulheres ${ }^{13}$.

Quanto à rede de apoio, 70 (23,7\%) alunos continuam morando com os pais, $148(50,2 \%)$ alunos moram em repúblicas e 77 $(26,1 \%)$ moram sozinhos. 
A maioria dos estudantes que não mora com a família opta por formar as chamadas repúblicas estudantis. O convívio com pessoas, na maioria das vezes recém-conhecidas, e a companhia dos colegas são importantes para superar a distância dos familiares e amigos. Esta característica é corroborada em um informe de uma universidade estadual ${ }^{14}$.

Em relação ao conhecimento sobre os programas de apoio da universidade, constatou-se que a maioria, 204 (69,1\%) acadêmicos, desconhece qualquer um deles. Somente 91 (30,9\%) conhecem algum deles, sendo que, destes, $72(24,4 \%)$ têm conhecimento de apenas um programa e $19(6,4 \%)$ têm ciência de mais de um. Entre os alunos que conhecem os programas, 5 $(1,7 \%)$ têm informações sobre o PAE, outros 56 (19,0\%) conhecem o Apoio Psicopedagógico, oferecido pela disciplina de Psiquiatria, 11 (3,7\%) têm ciência do Projeto Tutoria, 15 (5,1\%) conhecem o Apoio Psicopedagógico e o Projeto Tutoria, e somente $4(1,4 \%)$ conhecem todos os programas oferecidos pela universidade.

Isso pode refletir uma baixa divulgação dos programas ou pouco interesse dos alunos pelas atividades realizadas na instituição. O programa de ajuda mais conhecido é o Apoio Psicopedagógico, oferecido no Departamento de Medicina, o que sugere provável desinteresse dos acadêmicos por programas oferecidos fora do campus ou maior acessibilidade física dentro do próprio Departamento.

Entre os que conhecem os programas, $39(42,8 \%)$ procuraram alguma ajuda, 13 (33,4\%) escolheram psicólogos/psiquiatras particulares ou de convênio, 4 (10,2\%) procuraram ajuda da disciplina de Psiquiatria, 2 (5,1\%) o Projeto Tutoria, e 8 (20,6\%) mais de um programa de apoio.

Apenas 13,2\% do total de entrevistados procuraram alguma ajuda. Isto poderia ser correlacionado com aspectos como confidencialidade, custo, medo do estigma de desequilíbrio emocional, de admitir suas falhas ou dificuldades no aprendizado, além de preocupações em ser ajudado por um futuro colega ${ }^{10}$.

A assistência médica informal entre colegas faz parte da cultura médica e talvez reflita algo que os estudantes de Medicina captem de seus modelos profissionais. Sabe-se que estudantes de Medicina de fato aprendem posturas e valores a partir de exemplos de seus preceptores e que este aprendizado tem importância ao longo de sua vida ${ }^{15}$.

Grupos religiosos foram citados por $12(30,7 \%)$ dos que procuraram ajuda, o que coincide com um estudo qualitativo no qual os estudantes relataram a religiosidade como ajuda no enfrentamento dos problemas durante o curso ${ }^{4}$ (Tabela 1).
Tabela 1

Distribuição dos alunos que procuraram algum apoio psicológico

\begin{tabular}{lcc}
\hline Variável & $\mathrm{n}=39$ & $\%$ \\
\hline Psicólogo/Psiquiatra & 13 & 33,4 \\
Projeto Tutoria & 2 & 5,1 \\
Disciplina de psiquiatria & 4 & 10,2 \\
Mais de um programa & 8 & 20,6 \\
Grupos religiosos & 12 & 30,7 \\
\hline
\end{tabular}

A procura por cuidados foi, em sua maioria, espontânea - 18 $(6,1 \%) ; 2(0,7 \%)$ alunos foram encaminhados por professores/coordenador/chefe do Departamento, $4(1,4 \%)$ por seus pais, $1(0,3 \%)$ foi orientado por outro familiar e $2(0,7 \%)$ foram conduzidos por amigos. Isto se assemelha ao encontrado numa universidade estadual, em que 6,5\% dos alunos matriculados procuraram espontaneamente a ajuda do $\mathrm{Grapal}^{10}$. Entretanto, difere dos resultados expostos pelo Dr. Hamer Palhares Alves, coordenador da rede de apoio à Médicos da Escola Paulista de Medicina citado por Fagnani et al. (2005), onde 70\% foram encaminhados pela família, $15 \%$ por colegas e $15 \%$ buscaram voluntariamente ajuda ${ }^{16}$.

Dos acadêmicos que fazem uso de algum tipo de medicação - 62 (21,0\%) -, a maioria é do sexo feminino: 35 (56,4\%). Maior prevalência no sexo feminino também foi observada por Contee Gonçalves ${ }^{17}$, num estudo em que mulheres mencionam maior consumo de remédios, sobretudo devido à maior utilização de drogas que atuam no sistema endócrino, nutricional e metabólico.

Alguns fatores de risco conhecidos entre estudantes de Medicina para uso de substâncias psicotrópicas são: acesso fácil aos medicamentos; história familiar de dependência; problemas emocionais; estresse no trabalho e em casa; busca de emoções fortes; autoadministração no tratamento para dor e para o humor; fadiga crônica ${ }^{16}$.

Com relação às classes de medicamentos, os mais utilizados entre os alunos foram os antidepressivos - $22(35,4 \%)$ - e os ansiolíticos - 18 (29,0\%) (Tabela 2).

Entre os usuários, apenas 25 (40,3\%) procuraram algum tipo de cuidado. Dos que não procuraram ajuda, $9(24,3 \%)$ fazem uso de antidepressivos, $3(8,1 \%)$ de indutores do sono, 5 (13,5\%) de ansiolíticos e 4 (10,8\%) de inibidores do apetite. 
Tabela 2

Distribuição dos alunos que fazem uso de algum tipo de medicamento

Utilizando o questionário $S R Q-20$, encontramos na primeira série $10(14,7 \%)$ alunos com pontuação acima de 7; na segunda série, 24 (28,9\%); na terceira série, 22 (30,1\%); e na quarta série, 21 $(29,6 \%)$. No total, 77 (26,1\%) alunos obtiveram escore positivo. Facundes e Ludermir ${ }^{18}$ observaram em um estudo numa universidade federal prevalência maior de TPMe, de $42,6 \%$, entre estudantes de Medicina.

A média dos valores de escore encontrados foi 5,4 e a mediana 5,0 , sendo que o valor mínimo encontrado foi 0 (zero) e o máximo foi 17 .

\section{Análise univariada}

Entre os acadêmicos considerados com TPMe (escore acima de 7), 57 (75,0\%) eram do sexo feminino. No entanto, seis alunos não responderam ao item gênero e, entre eles, um apresentou escore positi-

\begin{tabular}{lcc}
\hline Variável & $\mathrm{n}=62$ & $\%$ \\
\hline Antidepressivos & 22 & 35,4 \\
Ansiolíticos & 18 & 29,0 \\
Antipsicóticos & 2 & 3,2 \\
Relaxantes musculares & 8 & 13,0 \\
Indutores do sono & 8 & 13,0 \\
Inibidores do apetite & 4 & 6,4 \\
\hline
\end{tabular}

vo. Outros artigos da literatura confirmam maior incidência no sexo feminino.

Em artigo publicado no Rio de Janeiro sobre estresse entre médicos residentes, são descritas evidências de que tanto médicas formadas quanto estudantes de Medicina do sexo feminino experimentam mais desconfortos psicológicos, como solidão, depressão, suicídio e conflitos conjugais ${ }^{6}$. Entretanto, um estudo feito por uma universidade estadual mostrou que entre seus alunos há equilíbrio entre o percentual de homens e mulheres com sintomas depressivos ${ }^{8}$.

A maioria dos alunos que tem TPMe-32 (41,6\%) - mora sozinha, enquanto a prevalência entre aqueles que moram com os pais ou em república foi muito semelhante: $15(21,4 \%)$ e 29 $(19,6 \%)$, respectivamente. Isto ocorre provavelmente porque o apoio familiar atua como suporte das circunstâncias estressantes relacionadas à graduação médica. No caso dos estudantes que optam por formar repúblicas, a presença dos colegas substitui em parte o acolhimento familiar, podendo diminuir a prevalência de transtornos neste grupo ${ }^{19}$ (Tabela 3).

Entre os alunos sem transtornos, 159 (72,6\%) não têm conhecimento dos programas oferecidos pela universidade. Já entre os acadêmicos com TPMe, aproximadamente $60 \%$ não conhecem qualquer programa, o que pode retardar a busca por ajuda. Observamos que 31 (40,8\%) indivíduos que conhecem ao menos um programa apresentam transtornos.

Entre os alunos que usam medicamentos, 32 (51,6\%) apresenta TPMe. No entanto, $30(48,4 \%)$ alunos sem transtornos utilizam algum tipo de medicamento.

Tabela 3

Distribuição dos alunos entrevistados quanto ao sexo† e moradia em relação a presença deTPMe

\begin{tabular}{|c|c|c|c|c|c|c|c|}
\hline \multirow{2}{*}{ Variáveis } & \multicolumn{2}{|c|}{ Com transtornos } & \multicolumn{2}{|c|}{ Sem transtornos } & \multicolumn{2}{|c|}{ Total } & \multirow{2}{*}{$\begin{array}{c}\text { Teste de } \\
\text { significância }\end{array}$} \\
\hline & $\mathrm{n}$ & $\%$ & $\mathrm{n}$ & $\%$ & $\mathrm{n}=289$ & $\%$ & \\
\hline Masculino & 19 & 15,7 & 102 & 84,3 & 121 & 100 & $X_{(1 ; 0,05)}^{2}=$ \\
\hline \multirow[t]{2}{*}{ Feminino } & 57 & 33,9 & 111 & 66,1 & 168 & 100 & $12,05^{*}$ \\
\hline & $\mathrm{n}$ & $\%$ & $\mathrm{n}$ & $\%$ & $\mathrm{n}=295$ & $\%$ & \\
\hline Com os pais & 15 & 21,4 & 55 & 78,6 & 70 & 23,7 & \multirow{3}{*}{$\begin{array}{c}X_{(2 ; 0,05)}^{2}= \\
13,67^{*}\end{array}$} \\
\hline República & 29 & 19,6 & 119 & 80,4 & 148 & 50,2 & \\
\hline Sozinho & 32 & 41,6 & 45 & 58,4 & 77 & 26,1 & \\
\hline
\end{tabular}

†Seis alunos não responderam à pergunta sobre gênero.

*Significativo. 
É elevado o número de estudantes que utilizam algum medicamento, mas sem apresentar TPMe. Segundo artigo publicado pela Australian Medical Association, após entrevista com 358 médicos, $71 \%$ destes se sentem envergonhados em procurar ajuda de colegas e $25 \%$ consideram aceitável a automedicação ${ }^{15}$. Estes resultados fortalecem a hipótese de que muitos alunos, além de se autodiagnosticarem, tomam condutas para suas

\section{Análise bivariada}

Foi verificado que, entre os 91 acadêmicos que conhecem os programas, 20 (30,8\%) apresentam transtornos e não procuraram qualquer tipo de ajuda, o que demonstra baixa eficiência dos programas da instituição e baixa procura por programas alternativos. Por outro lado, a procura, quando do conhecimento do sistema de ajuda, com ou sem TPMe, foi universal.

Tabela 4

Distribuição dos alunos entrevistados quanto ao uso de medicamentos em relação à prevalência de TPMe

\begin{tabular}{|c|c|c|c|c|c|c|c|}
\hline \multirow[t]{2}{*}{ Variável } & \multicolumn{2}{|c|}{ Com transtornos } & \multicolumn{2}{|c|}{ Sem transtornos } & \multicolumn{2}{|c|}{ Total } & \multirow[t]{2}{*}{$\begin{array}{c}\text { Teste de } \\
\text { significância }\end{array}$} \\
\hline & $\mathrm{n}=83$ & $\%$ & $\mathrm{n}=212$ & $\%$ & $\mathrm{n}=295$ & $\%$ & \\
\hline Usa & 32 & 51,6 & 30 & 48,4 & 62 & 100 & $\begin{array}{c}X^{2}{ }_{(1 ; 0,05)}= \\
21,39^{*}\end{array}$ \\
\hline Não usa & 51 & 21,9 & 182 & 88,1 & 233 & 100 & \\
\hline
\end{tabular}

*Significativo

supostas condições clínicas (Tabela 4).

Correlacionando as respostas do $S R Q-20$ com as do questionário desenvolvido pelos autores, foi encontrada significância estatística entre TPMe e as seguintes condições: sexo feminino, morar sem a família e uso de medicação (psicotrópica).

Não foi possível concluir se a parcela da amostra sem transtornos que procurou ajuda se apresenta nesta condição porque o fez precocemente ou se estes alunos nunca apresentaram quaisquer tipos de TPMe (Tabela 5).

Tabela 5

Distribuição dos alunos entrevistados quanto ao conhecimento dos programas de apoio em relação à prevalência de TPMe e a procura por cuidados

\begin{tabular}{|c|c|c|c|c|c|c|c|c|c|c|}
\hline \multirow{3}{*}{ Variáveis } & \multicolumn{4}{|c|}{ Com transtornos } & \multicolumn{4}{|c|}{ Sem transtornos } & \multirow{2}{*}{\multicolumn{2}{|c|}{ Total }} \\
\hline & \multicolumn{2}{|c|}{ Procurou } & \multicolumn{2}{|c|}{ Não procurou } & \multicolumn{2}{|c|}{ Procurou } & \multicolumn{2}{|c|}{ Não procurou } & & \\
\hline & $\mathrm{n}=12$ & $\%$ & $\mathrm{n}=65$ & $\%$ & $\mathrm{n}=15$ & $\%$ & $\mathrm{n}=203$ & $\%$ & $\mathrm{n}=295$ & $\%$ \\
\hline Conhece & 12 & 100 & 20 & 30,8 & 15 & 100 & 44 & 21,7 & 91 & 30,9 \\
\hline Não conhece & 0 & 0 & 45 & 69,2 & 0 & 0 & 159 & 78,3 & 204 & 69,1 \\
\hline $\begin{array}{c}\text { Teste de } \\
\text { significância }\end{array}$ & \multicolumn{4}{|c|}{$\mathrm{X}^{2}{ }_{(1 ; 0,05)}=11,19^{*}$} & \multicolumn{6}{|c|}{$X_{(1 ; 0,05)}^{2}=29,57^{*}$} \\
\hline
\end{tabular}

*Significativo. 
O uso de medicamentos entre alunos com transtornos é duas vezes maior no sexo feminino. Além disso, a prevalência do uso de algum tipo de medicamento entre os acadêmicos sem transtornos é muito semelhante entre os sexos.

Segundo Meleiro ${ }^{20}$, o perfil psicológico que leva as mulheres a escolherem a carreira médica é: serem competitivas, ambiciosas, compulsivas, individualistas e inteligentes. Não podemos inferir se o maior uso de medicamentos entre as acadêmicas se dá em função deste perfil psicológico predominante ou devido a maior prevalência de TPMe neste grupo.

A análise estatística demonstrou significância entre TPMe e as seguintes condições: conhecimento de programas e ter procurado assistência.

\section{CONCLUSÕES}

Concluiu-se que a prevalência de TPMe entre estudantes de Medicina da primeira à quarta série é de 26,1\%. Observou-se maior acometimento do sexo feminino e menor prevalência na primeira série, enquanto as outras apresentam percentuais semelhantes.

Foi possível concluir também que há grande desconhecimento dos graduandos, sendo que $69,1 \%$ não conhecem nenhum programa de apoio. O programa mais conhecido é o Apoio Psicopedagógico, oferecido pela disciplina de Psiquiatria.

Houve também baixa procura por cuidados entre os acadêmicos $(9,1 \%)$. O tipo de ajuda mais procurado foi o de psicólogos e psiquiatras não vinculados à instituição, sendo que esta procura foi, em sua maioria, espontânea.

\section{CONSIDERAÇÕES FINAIS}

A alta prevalência dos TPMe, bem como a falta de conhecimento e baixa procura por ajuda dos estudantes de Medicina mostram a necessidade de maior divulgação e efetividade dos programas de apoio psicopedagógicos oferecidos ao aluno pela instituição.

Os resultados obtidos neste estudo possibilitam realizar novas pesquisas, a fim de obter informações mais aprofundadas sobre as variáveis, como, por exemplo, tipos de medicamentos usados pelos acadêmicos e formas de aquisição dos mesmos, uma vez que a maioria dos alunos afirmou fazer uso de medicamentos sem ter procurado ajuda médica. Novas pesquisas sobre o uso de drogas lícitas e ilícitas como forma de enfrentar o estresse gerado durante a graduação possibilitarão alcançar conclusões mais específicas sobre o tema.

Os resultados foram apresentados à coordenação pedagógica do curso de Medicina e à chefia de Departamento.

\section{REFERÊNCIAS}

1. Universidade de Taubaté. Pró-reitorias. Pró-reitoria Estudantil. Programa de Apoio ao Estudante [online]. Taubaté: UNITAU ; 2007 [acesso em 15 mai. 2007]. Disponível em: http:/ / www.unitau.br/universidade/pro-reitorias/estudantil-pre/projeto-pae/?searchterm=PAE

2. Organização Pan-Americana da Saúde, Organização Mundial da Saúde. Relatório sobre a Saúde do Mundo2001 Saúde mental: nova concepção, nova esperança. [documento na internet]. Organização Mundial da Saúde; 2001. [acesso em 15 mai. 2007]. Disponível em: www.dgs.pt/upload/membro.id/ficheiros/i006020.pdf

3. Castro FC. Os Temores na Formação e Prática da Medicina: Aspectos Psicológicos. Rev Bras Educ Med. 2004;28(1):38-45.

4. Zonta R, Robles AC, Grosseman S. Estratégias de enfrentamento do estresse desenvolvidas por estudantes de Medicina da Universidade Federal de Santa Catarina. Rev Bras Educ Med. 2006;30(3):147-53.

5. Moro A, Valle J, Lima L. Sintomas Depressivos nos Estudantes de Medicina da Universidade da Região de Joinville (SC). Rev Bras Educ Med. 2005;29(2):97-102.

6. Costa L, Pereira CA. O Abuso como Causa Evitável de Estresse entre Estudantes de Medicina. Rev Bras Educ Med. 2005;29(3):185-90.

7. Almeida-Filho N, Mari JJ, Coutinho ESF. Migração, inserção produtiva e saúde mental na modernidade tardia: novas evidências do estudo multicêntrico de morbidade psiquiátrica em áreas metropolitanas brasileiras. Rev Psiquiatr Clín (São Paulo). [periódico na Internet]. 1999. [acesso em 15 maio 2007];26(5).

Disponível em: http://www.hcnet.usp.br/ipq/revista/index.html

8. Porcu M, Fritzen CV, Helber C. Sintomas depressivos nos estudantes de medicina da Universidade Estadual de Maringá. Psiquiatr Prat Med (São Paulo) [periódico na Internet]. 2001 [acesso em 17 maio 2007]; 34 (1).Disponível em: http://www.unifesp.br/dpsiq/polbr/ppm/original5_01.htm

9. Majella R. Cuidando de quem cuida. Jornal da Paulista [periódico na Internet]. 2002 [acesso em 20 maio 2007].

Disponível em: http://www.unifesp.br/comunicacao/jpta/ed165/mercado.htm

10. Millan LR, Arruda PCV. Assistência Psicológica ao estudante de medicina: 21 anos de experiência. Rev Assoc Med Bras. 2008;54(1):90-4. 
11. Harding TW, Arango MV, Baltazar J, Climent CE, Ibrahim HHA, Ignácio LL, et al. Mental Disorders in primary health care: a study of their frequency and diagnosis in four development countries. Psychol Med. 1980;10:231-41.

12. Ludermir AB. Inserção produtiva, gênero e saúde mental. Cad Saúde Pública. 2000;16(3): 647-59.

13. Zanella J. Amplo perfil. Jornal da Unesp [periódico na Internet]. 2003 ago [acesso em 19 jun. 2007]; 180. Disponível em http://www.unesp.br/aci/jornal/180/alunos.htm

14. Pontifícia Universidade Católica - Minas. Como os estudantes da PUC Minas Betim lidam com a liberdade e a responsabilidade de morar em repúblicas. PUC Informa Betim [periódico na Internet]. 2003 mar [acesso em 19 jun. 2007]; 29. Disponível em: http://www.pucminas.br/informativo/puc_informa_lista.php.

15. Brimstone R, Thistlethwaite JE, Quirk F. Behaviour of medical students in seeking mental and physical health care: exploration and comparison with psychology students. Med Educ [periódico na Internet]. 2007. [acesso em 13 jun. 2007]; 41(1). Disponível em: http:/ /www.blackwell-synergy.com/doi/abs/10.1111/j.1365-2929.2006.02649.x

16. Fagnani C, Maeda D, Guijt M. Como anda a saúde do médico brasileiro? Associação Brasileira de Psiquiatria [on line]. 2005. [acesso 13 jun. 2007]. Disponível em: http:/ / www.abpbrasil.org.br/imprensa/release/exibRelease $/$ ?release $=9$

17. Conte M, Gonçalves A. Ampliando Elementos da Educação Médica: Morbidade Referida em Universitários de Medicina. Rev Bras Educ Med [periódico na Internet]. 2006. [acesso em 13 jun. 2007]; 30(1). doi:10.1590/s0100-55022006000100003.

18. Facundes VLD, Ludermir AB. Common mental disorders among health carestudents. Rev Bras Psiquiatr [periódicona Internet]. 2005. [acesso em 14 jun 2007); 27(3). doi: 10.1590/s1516-44462005000300007.

19. Gaviria S, Rodrigues MA, Alvarez T. Calidad de la relación familiar y depresión en estudiantes de medicina de Medellín. Rev Chil Neuro-Psiquiatr [periódico na Internet]. 2002. 40(1). [acesso em 13 jun. 2007]; 41-6. doi: 10.4067/s0717-92272002000100005.

20. Meleiro AMAS. Suicídio entre médicos e estudantes de medicina. Rev Assoc Med Bras. 1992;44(2):135-40.

\section{APÊNDICE E ANEXO}

Os instrumentos utilizados estão disponíveis para consulta em http://www.educacaomedica.org.br/anexos

\section{CONTRIBUIÇÃO DOS AUTORES}

A elaboração do projeto de pesquisa, coleta e interpretação de dados, análise descritiva dos resultados, conclusões e revisão bibliográfica foram feitas por Ágnes de Paula Fernandes Watanabe, Antonio Augusto de França Neves, Carolina Cruz Furtado Ribeiro, Fernanda Jacopucci Hehn, Marco Antonio Buch Cunha e Tatiana Paludetto Lopes. Por se tratar de uma pesquisa realizada em grupo durante as aulas da disciplina de Saúde Coletiva, sob orientação da Professora Doutora Maria Elisa Moreira, todos os integrantes tiveram igual dedicação e participação.

A análise estatística foi desenvolvida por Marco Antonio Buch Cunha.

As alterações necessárias à publicação e correções foram realizadas por Antonio Augusto de França Neves e Marco Antonio Buch Cunha.

Todo o estudo foi elaborado, interpretado e redigido sob orientação da Professora Doutora Maria Elisa Moreira.

\section{CONFLITO DE INTERESSES}

Declarou não haver

\section{ENDEREÇO PARA CORRESPONDÊNCIA}

Marco Antonio Buch Cunha

Avenida Tiradentes, 477 - apto 82

Jardim das Nações - Taubaté

CEP.: 12030-180 SP

E-mail: marcoabc@gmail.com 